\title{
CARACTERIZACIÓN DEL CONSUMO DE HORTALIZAS EN LAS FAMILIAS DEL SUR-SUR DE COSTA RICA ${ }^{1}$
}

\author{
Alexis Villalobos-Monge², Luis Sánchez-Chacón ${ }^{3}$
}

\section{RESUMEN}

Caracterización del consumo de hortalizas en las familias del sur-sur de Costa Rica. El objetivo de este trabajo fue determinar los diferentes aspectos cuantitativos y cualitativos que explican la cultura de consumo actual de hortalizas en las familias residentes en la zona sur-sur de Costa Rica. Este trabajo expone resultados relacionados a la caracterización realizada en el 2011 en familias residentes en la denominada zona sur-sur de Costa Rica sobre la cultura de consumo de productos hortícolas. Se aplicaron cuestionarios a una muestra estratificada de familias; lo que permitió establecer la valoración de los precios de mercado, por parte de las familias, para consumir estos alimentos, donde se determinó un valor máximo de US\$1,74 millones por semana. Los principales rubros de consumo de acuerdo al valor pagado fueron el tomate, la papa, el plátano, la cebolla y el brócoli, para citar los cinco principales. También fue posible establecer valoraciones cualitativas sobre el consumo de estos productos; por ejemplo se determinó que para el 71,2\% de las familias, la frescura representa la característica de mayor valor, además, el principal sitio donde los núcleos familiares realizan las compras de estos alimentos corresponde a supermercados $(38,4 \%$ de los casos $)$.

Palabras clave: cultura hortícola, aspectos cuantitativos del consumo, aspectos cualitativos del consumo.

\begin{abstract}
Characterization of consumption of vegetables in the families of the south-south of Costa Rica. The objective of this work was to determine the various quantitative and qualitative aspects that explain the culture of vegetable consumption of families living in the so-called south-south region of Costa Rica. This work presents results from the characterization of horticulture products consumption culture performed in 2011 amongst families that live in the region known as south-south Costa Rica. Through surveys applied to a stratified sample of families, market prices were determined for home consumption of these foods, which determined a maximum value of US\$1,74 million per week, where the main items of consumption according to the amount paid would be the tomato, potato, plantain, onion and broccoli to name the top five. Qualitative assessments of the consumption of these products were also possible to establish, for example, $71.2 \%$ of families in the study area indicated that freshness was the most valuable property for purchase, amongst other traits such as the main place to acquire these foods, were the supermarket was the most common corresponding to $38.4 \%$ of cases.
\end{abstract}

Key words: horticultural consumption, quantitative aspects, qualitative aspects.

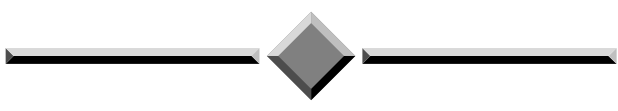

\footnotetext{
1 Recibido: 8 de agosto, 2012. Aceptado: 1 de abril, 2013. Resultados parciales del proyecto 736-A7-059: Investigación de mercados para productos agrícolas, Vicerrectoría de Investigación, Universidad de Costa Rica.

2 Estación Experimental Agrícola Fabio Baudrit Moreno, Universidad de Costa Rica. Alajuela, Costa Rica. Apdo. 183-4050, Alajuela, Costa Rica. alexis.villalobosmonge@ucr.ac.cr

3 Centro de Investigación en Economía Agrícola y Desarrollo Agroempresarial, Universidad de Costa Rica. San José, Costa Rica. lalfonso2385@hotmail.com
}

(C) 2013 Agronomía Mesoamericana es desarrollada en la Universidad de Costa Rica y se encuentra licenciada con Creative Commons Reconocimiento-NoComercial-SinObraDerivada 3.0 Costa Rica. Para más información escríbanos a pccmca@ucr.ac.cr 


\section{INTRODUCCIÓN}

Desde el siglo XX se ha profundizado el interés por conocer las motivaciones que impulsan el consumo por determinados bienes y servicios. Estas particularidades las han estudiado varias ramas científicas y en la actualidad predomina un enfoque interdisciplinario para estos abordajes, todo en virtud de manejar criterios más comprensivos que permitan explicar adecuadamente las razones que promueven una determinada decisión de consumo en las poblaciones humanas. Esto es lo que autores como Solomon (2008) denominan comportamiento del consumidor en un sentido amplio.

Este comportamiento se encuentra matizado por una serie de características implícitas al tipo de producto o servicio, donde resulta conveniente el tratamiento que autores como Chiavenato (2000) emplean para explicar las maneras en que un individuo decide satisfacer sus diferentes necesidades. La alimentación es parte de los aspectos de tipo fisiológico que deben satisfacer las poblaciones y uno de los elementos que actualmente aporta a esta discusión es que los alimentos que se consumen deben cumplir con dos premisas fundamentales: ser originados considerando la calidad como eje transversal en todo el proceso de producción, y no atentar de ninguna forma contra la salud humana, lo cual forma parte de los postulados que promulga y fomenta la Organización de las Naciones Unidas (ONU) a través de la comisión del Codex Alimentarius (FAO 2012) y se discute en los foros internacionales colocando especial énfasis en aspectos como las formas en que la globalización y el comercio mundial moldean precisamente estos nuevos paradigmas (Nicola 2010).

Partiendo de estas premisas cabe preguntarse lo siguiente: ¿Cuáles características implican la dimensión de la calidad y deben ser consideradas para que un alimento satisfaga adecuadamente las necesidades de un grupo de personas?. Para argumentar una respuesta amplia se debe pasar por varios filtros científicos y entre ellos la investigación de mercados es uno de significativa relevancia.

En ese sentido, existen planteamientos teóricos (Kotler y Armstrong 2003) que sugieren que para entender la manera en que un grupo de personas responde a estímulos (como la calidad) se deben observar en detalle los aspectos cuantitativos y cualitativos de la demanda por el producto o servicio. Es por lo anterior, que en este trabajo se discuten estos elementos del mercado, buscando apoyar los esfuerzos que se han desarrollado en los últimos años, en la Estación Experimental Agrícola Fabio Baudrit Moreno (EEAFBM) de la Universidad de Costa Rica, en el sentido de entender la dinámica del mercado hortícola en Costa Rica, con el fin de promover proyectos locales que atiendan las oportunidades y necesidades originadas principalmente por el entorno regional.

La población de los cantones Buenos Aires, Coto Brus, Osa, Golfito y Corredores, todos pertenecientes a la provincia de Puntarenas y denominados dentro de este trabajo como "sur-sur", han sido objeto de estudio para conocer con mayor nivel de detalle la dinámica del consumo de hortalizas existente en esta región del país, para la definición de políticas efectivas para una región que ha obtenido históricamente bajas puntuaciones en los Índices de Desarrollo Social, medidos por distintos organismos como el Ministerio de Planificación Nacional y Política Económica (MIDEPLAN 2007) y el Programa de las Naciones Unidas para el Desarrollo Humano (PNUD 2006).

El objetivo de este trabajo fue determinar los diferentes aspectos cuantitativos y cualitativos que explican la cultura de consumo actual de hortalizas en el grupo humano que conforman las familias residentes en la zona sur-sur de Costa Rica.

\section{MATERIALES Y MÉTODOS}

Para la realización del presente trabajo se consideraron familias residentes en los cantones de Buenos Aires, Osa, Corredores, Coto Brus y Golfito, todos pertenecientes a la provincia de Puntarenas. Se elaboró un cuestionario para conocer los elementos relacionados al proceso de decisión sobre el consumo semanal de hortalizas por parte de este grupo. El cuestionario implicó 150 variables de consulta y se aplicó en el periodo comprendido entre los meses de abril a agosto del 2011. Las variables de interés se agruparon de la siguiente forma:

1. Información general: en este grupo de variables se incluyeron aspectos como género, edad, nombre, total de adultos y niños que viven en la casa, total de miembros que aportan dinero a la casa, 
gasto familiar por semana en alimentos, teléfono de contacto, lugar de residencia y correo electrónico.

2. Consumo semanal que la familia realiza por tipo de hortaliza: entre las variables que fueron consultadas para este grupo estaba el valor aproximado que semanalmente consumía la familia para un listado de 41 hortalizas, esto de acuerdo a la presentación comercial que se consiguiera en el sitio de compra. También se consultó por el orden de preferencia entre varias alternativas de sitios de compra de productos hortícolas.

3. Características cualitativas de importancia para las hortalizas consumidas: en este tercer grupo de variables se consultó sobre el orden de importancia respecto a un listado de características que las hortalizas consumidas debían cumplir según las necesidades de la familia, también sobre si las características anteriores las encontraban en los sitios de compra habituales de estos alimentos. Otras variables consideradas fueron los días y frecuencia semanal de compra, así como una consulta para conocer el cambio probable en los hábitos de consumo si las características de mayor relevancia para la familia se presentaran de forma consistente en los sitios de compra.

4. Información sobre otras características de las hortalizas consumidas: finalmente se agruparon variables para conocer otros aspectos relacionados al consumo habitual de hortalizas como la preferencia de consumir estas con algún grado de procesamiento, el conocimiento sobre el origen de los productos hortícolas consumidos así como estimar la probable preferencia de consumir hortalizas producidas en la zona sur de Costa Rica en comparación a otros sitios.

En este trabajo se discuten únicamente los resultados de las variables contenidas en el segundo y tercer grupo.

Una vez que se validó el cuestionario por medio de una prueba piloto, la cual consistió en aplicar a un subgrupo de jefes y jefas de familia la versión preliminar del cuestionario que finalmente se utilizaría en el trabajo de campo con el fin de ajustar el tipo de pregunta (abierta, cerrada o mixta) así como la duración promedio en completar los cuestionarios por parte de cada persona. Como resultado de esta prueba se obtuvo un total de 23 cuestionarios que fueron aplicados a personas residentes principalmente de los cantones de Buenos Aires y Golfito, esto por conveniencia para los autores en la atención de reuniones programadas en estos lugares. Se procedió a tabular la información y obtener los promedios de los tiempos de duración por persona, lo cual facilitó el establecimiento de factores relacionados con la logística y la duración del trabajo de campo por llevar a cabo. Con los resultados del proceso anterior se determinó el tamaño de muestra, empleando para ello un diseño de muestreo estratificado, donde las residencias por cantón representaron las unidades estadísticas convenientes. Se empleó la metodología definida por Gómez (Gómez 1998) para establecer el tamaño de muestra:

Donde:

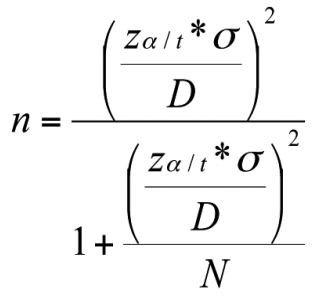

$\mathrm{n}=$ Tamaño de la muestra .

$z \alpha=$ Probabilidad de ocurrencia del valor $\mathrm{z}$ para un nivel de confianza $\alpha$ para una población con distribución normal.

$\sigma=$ Desviación estimada de la población.

$\mathrm{D}=$ Error de estimación asociado al nivel de confianza requerido.

$\mathrm{N}$ = Tamaño de la población

Para desarrollar la expresión anterior fue necesario utilizar información elaborada por el Instituto Nacional de Estadísticas y Censos (INEC) respecto a la cantidad de viviendas existentes en la zona de estudio. La información disponible a la fecha de ejecución del trabajo de campo permitió establecer un total de 50 380 viviendas (INEC 2000), las cuales conformaron las unidades estadísticas para la recolección de información e inferencia de resultados (Cuadro 1).

Desarrollando la expresión y al asumir los siguientes valores:

$z \alpha / t=1,96$

$\sigma=8$ (variación en el consumo en unidades, kilogramos o rollos por semana por vivienda) ${ }^{4}$

4 Se asumió un valor de 8 para la variable $\sigma$ como el más adecuado en función de experiencias anteriores desarrolladas en la zona de estudio por parte del autor principal. 
Cuadro 1. Viviendas existentes en los cantones de Buenos Aires, Osa, Golfito, Coto Brus y Corredores, provincia de Puntarenas, Costa Rica. Periodo del 1 de abril al 31 agosto de 2011 .

\begin{tabular}{lc}
\hline Cantón & Viviendas \\
\hline Buenos Aires & 10182 \\
Osa & 7496 \\
Golfito & 10006 \\
Coto Brus & 12224 \\
Corredores & 10472 \\
Total & 50380 \\
\hline
\end{tabular}

Fuente: INEC (2000).

$\mathrm{D}=1$ (error de estimación en unidades, kilogramos o rollos por semana)

$\mathrm{N}=50380$ viviendas

Se obtuvo un valor para $n=245$ viviendas; debido a que no se contaba con los recursos humanos y financieros suficientes. No obstante, para ejecutar el trabajo de campo se decidió asumir un ajuste de hasta un $50 \%$ para mejorar la inferencia que se realizaría de la información recolectada en el campo, por tanto se trabajó un tamaño final de muestra de 367 viviendas, las cuales se asignaron según se aprecia en el Cuadro 2.

En el trabajo de recolección de los datos se realizó en cuatro giras a la zona, donde se obtuvo un total de 393 cuestionarios completos, un 7\% adicional al tamaño de muestra definido inicialmente. La encuesta se aplicó en los principales núcleos urbanos de los cantones descritos (Cuadro 2), a la persona encargada de definir o realizar la compra de hortalizas para cada vivienda consultada las cuales fueron elegidas al azar.

En el cantón de Buenos Aires se aplicaron 84 cuestionarios en el distrito homólogo. Para el caso del cantón de Osa, se distribuyó la muestra en tres distritos: Ciudad Cortés (20 cuestionarios), Palmar Norte (32 cuestionarios), y Sierpe (13 cuestionarios). El cantón de Corredores tuvo dos distritos de enfoque: Corredor o Ciudad Neilly (47 cuestionarios) y Paso Canoas (23 cuestionarios). En el caso de Coto Brus se aplicaron cuestionarios en tres distritos: San Vito (47 cuestionarios), Sabalito (35 cuestionarios) y Agua Buena (22 cuestionarios). Finalmente, el cantón de Golfito presenta dos distritos importantes según la
Cuadro 2. Asignación de la muestra para la realización del trabajo de campo para conocer los aspectos relacionados al consumo de hortalizas. Cantones de Buenos Aires, Osa, Golfito, Coto Brus y Corredores, provincia de Puntarenas, Costa Rica. Periodo del 1 de abril al 31 agosto de 2011.

\begin{tabular}{lc}
\hline Cantón & Tamaño de muestra por cantón \\
\hline Buenos Aires & 84 \\
Osa & 52 \\
Golfito & 70 \\
Coto Brus & 83 \\
Corredores & 78 \\
Total & 367 \\
\hline
\end{tabular}

densidad poblacional: Guaycará o Río Claro (36 cuestionarios) y Golfito (34 cuestionarios). La distribución de las submuestras en los respectivos distritos se basó en las proyecciones de las poblaciones según el INEC para el año 2011 (INEC 2011).

La información se organizó y tabuló por medio de hojas de cálculo en el programa Excel 2010 así como en el Paquete Estadístico SPSS versión 15. Para obtener las estimaciones poblacionales para la media y los límites de confianza al 95\% para el consumo de hortalizas, se emplearon las siguientes expresiones (Mendenhall et al. 2008):

$$
\bar{x}_{j}-1,96 * \frac{s_{j}}{\sqrt{n}} \leq \mu \leq \bar{x}_{j}+1,96 * \frac{s_{j}}{\sqrt{n}}
$$

Donde:

$\mu=$ Media del consumo semanal de la hortaliza $j$ por vivienda.

$\bar{x}=$ Media muestral del consumo semanal de la hortaliza $j$ por vivienda.

$s_{j}=$ Desviación estándar del consumo semanal de la hortaliza $j$ por vivienda.

$n=$ Total de viviendas incluidas en la muestra.

Finalmente la media poblacional para el consumo semanal de hortalizas $\left(\mu_{j}\right)$ se obtuvo de la siguiente expresión:

$$
\mu_{j}=N * p * \bar{x}_{j}
$$

Donde:

$\mu_{j}=$ Media poblacional del consumo semanal para la hortaliza $j$. 
$N=$ Total de viviendas consideradas en la población.

$p=$ Proporción de viviendas de la muestra que presentaron consumo semanal por la hortaliza $j$.

$\bar{x}_{j}=$ Media muestral del consumo semanal de la hortaliza $j$ por vivienda.

Para realizar la estimación del valor de mercado para las hortalizas consumidas semanalmente por la población de estudio, se consultó la base de datos del Programa Integral de Mercadeo Agropecuario (PIMA) referente a los precios en que fueron transadas las hortalizas (PIMA 2012a). Para ello se consultaron los precios modales que presentaron dichos alimentos en las 65 plazas mayoristas desarrolladas entre abril y agosto de 2011 en el Centro Nacional de Abastecimiento y Distribución de Alimentos (CENADA). Para efectuar el cálculo del rango semanal de valoración del mercado, se identificaron los valores máximos y mínimos encontrados en dicho periodo y se realizó el cálculo a partir de la siguiente expresión:

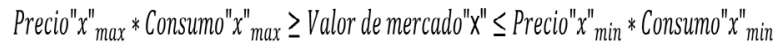

Donde:

Precio " $x{ }_{\text {max }}=$ Precio máximo para la hortaliza " $x$ " en el CENADA según la unidad de consumo familiar.

Consumo “ $x "{ }_{\max }=$ Valor de consumo semanal máximo para la hortaliza " $x$ " por parte de las familias de la zona de estudio.

Precio “ $x "{ }_{\min }=$ Precio mínimo para la hortaliza “ $\mathrm{x}$ " en el CENADA según la unidad de consumo familiar.

Consumo “ $x "{ }_{\min }=$ Valor de consumo semanal mínimo para la hortaliza " $x$ " por parte de las familias de la zona de estudio.

Los valores monetarios se presentan utilizando un tipo de cambio entre el colón costarricense (CRC) y el dólar estadounidense (US\$) de US\$1= CR $\$ 500$.

\section{RESULTADOS Y DISCUSIÓN}

Aspectos cuantitativos del consumo semanal de las familias residentes en la zona de estudio según el tipo de hortaliza

Los resultados encontrados para este grupo de variables, refleja que existen concordancias entre los productos hortícolas que consumen semanalmente las familias de la zona de estudio respecto a lo que han encontrado otras fuentes. En este sentido, el PIMA reporta entre las diez hortalizas de mayor preferencia por las familias costarricenses el tomate, la papa, el repollo, la zanahoria, la lechuga, el chayote, el chile dulce, la cebolla, el culantro castilla y la yuca (PIMA 2012b).

Para el caso de la población analizada en este trabajo, los resultados muestran que las diez hortalizas que consume semanalmente con más frecuencia la población de estudio son: la cebolla, la papa, el tomate, el chile dulce, el ajo, el plátano, la lechuga, el culantro castilla, el chayote y el apio (Cuadro 3). Se evidencia una concordancia para siete productos entre ambas fuentes de información respecto a la preferencia para consumir dichos alimentos.

En cuanto a las cantidades consumidas semanalmente por tipo de hortaliza, es importante destacar la existencia de hortalizas que son consumidas en mayor proporción que otras; en el primer grupo destacan el plátano, el chile dulce y el chayote mientras que entre las hortalizas de menor consumo se tiene al eneldo, los hongos frescos, los espárragos frescos y la alfalfa (Cuadro 4).

Para el caso de las diez hortalizas de mayor preferencia se estimó que tienen una media poblacional de consumo semanal por producto de $70576 \mathrm{~kg}$ de cebolla, $83771 \mathrm{~kg}$ de papa, $93852 \mathrm{~kg}$ de tomate, $151761 \mathrm{uni}-$ dades de chile dulce, 98598 cabezas de ajo, 320280 unidades de plátano, 79985 unidades de lechuga, 85079 rollitos de culantro castilla, 123248 unidades de chayote y finalmente, 46767 rollitos de apio.

Se puede apreciar que las cantidades consumidas son mayores para algunos productos que no presentan la intensidad en la frecuencia de consumo en virtud de lo mostrado en el Cuadro 3, razón por lo que se emplea la valoración monetaria de las cantidades consumidas, como otro parámetro que apoye la explicación de la dinámica en la cultura de consumo para estos alimentos.

Por tanto, la valoración a precios de mercado para el periodo de tiempo en que se efectuó el trabajo de campo, supone un valor semanal a precios de CENADA ubicado entre US\$1.020.774 y US\$361.991 para el caso de los 41 productos hortícolas considerados (Figura 1).

Se determinó que el tomate es la hortaliza que representa el mayor valor de mercado, esto por encontrarse un valor para la cantidad consumida por las familias de la zona de estudio cercano a los US\$219.000 
Cuadro 3. Clasificación de las hortalizas consumidas según la frecuencia semanal de consumo en los núcleos familiares de cinco cantones de Puntarenas, Costa Rica. Abril a agosto, 2011.

\begin{tabular}{|c|c|c|}
\hline $\begin{array}{l}\text { Puesto de } \\
\text { preferencia }\end{array}$ & Hortaliza & $\begin{array}{c}\text { Frecuencia sema- } \\
\text { nal de consumo }\end{array}$ \\
\hline 1 & Cebolla & $96,7 \%$ \\
\hline 2 & Papa & $95,2 \%$ \\
\hline 3 & Tomate & $94,7 \%$ \\
\hline 4 & Chile dulce & $92,6 \%$ \\
\hline 5 & Ajo & $90,1 \%$ \\
\hline 6 & Plátano & $85,8 \%$ \\
\hline 7 & Lechuga & $81,4 \%$ \\
\hline 8 & Culantro castilla & $78,9 \%$ \\
\hline 9 & Chayote & $76,6 \%$ \\
\hline 10 & Apio & $74,6 \%$ \\
\hline 11 & Pepino & $68,7 \%$ \\
\hline 12 & Repollo & $64,1 \%$ \\
\hline 13 & Zanahoria & $64,1 \%$ \\
\hline 14 & Yuca & $61,6 \%$ \\
\hline 15 & Culantro coyote & $61,1 \%$ \\
\hline 16 & Vainica & $57,3 \%$ \\
\hline 17 & Brócoli & $52,7 \%$ \\
\hline 18 & Ayote tierno & $51,4 \%$ \\
\hline 19 & Coliflor & $50,6 \%$ \\
\hline 20 & Remolacha & $41,2 \%$ \\
\hline 21 & Camote & $37,7 \%$ \\
\hline 22 & Ayote sazón & $32,8 \%$ \\
\hline 23 & Elote & $29,8 \%$ \\
\hline 24 & Ñampí & $29,5 \%$ \\
\hline 25 & Orégano & $27,0 \%$ \\
\hline 26 & Tiquizque & $26,7 \%$ \\
\hline 27 & Rábano & $22,6 \%$ \\
\hline 28 & Espinaca & $20,1 \%$ \\
\hline 29 & Cebollino & $14,5 \%$ \\
\hline 30 & Zapallo & $5,3 \%$ \\
\hline 31 & Minivegetales & $4,8 \%$ \\
\hline 32 & Albahaca & $3,8 \%$ \\
\hline 33 & Berenjena & $3,6 \%$ \\
\hline 34 & Zuquini & $3,3 \%$ \\
\hline 35 & Tomillo & $3,1 \%$ \\
\hline 36 & Elote dulce & $2,5 \%$ \\
\hline 37 & Alfalfa & $2,3 \%$ \\
\hline 38 & Perejil & $2,0 \%$ \\
\hline 39 & Espárrago & $1,8 \%$ \\
\hline 40 & Hongos & $1,0 \%$ \\
\hline
\end{tabular}

semanales en su precio máximo, seguido por hortalizas como la papa, el plátano, la cebolla y el brócoli.

La valoración anterior no contempla el respectivo margen de intermediación que existe entre una fuente mayorista de comercialización como el CENADA, respecto a los valores que finalmente paga el consumidor familiar en los sitios donde compra los alimentos. Se determinó un margen de intermediación de hasta un $70 \%$ respecto a los precios de referencia del CENADA, situación que ajustaría el valor de mercado para los productos en cuestión en un rango entre US\$1.735.316 y US\$615.385 por semana.

\section{Aspectos cualitativos del consumo semanal de las familias residentes en la zona de estudio según el tipo de hortaliza}

Ante la consulta sobre los principales sitios de compra el 38,4\% indicó que el principal sitio donde la familia compra las hortalizas corresponde a supermercados, seguido por las verdulerías con el 14,2\% (Cuadro 5).

Al comparar los resultados obtenidos con los citados por PIMA para las familias costarricenses (PIMA 2012), se observó que existe una diferencia considerable para el caso de la población familiar considerada, ya que para la zona de estudio las Ferias del Agricultor significan el tercer lugar de preferencia, mientras que en la información reportada por PIMA este canal de comercialización está ubicado en el primer lugar de las preferencias de las familias costarricenses. El 30\% de los consultados no respondieron esta pregunta, por lo que se deberá profundizar en posteriores investigaciones que se deriven en esta línea.

De manera adicional, se consultó sobre las principales características que eran valoradas para decidir sobre la realización de la compra de cada producto hortícola de preferencia. El 71,2\% de las familias entrevistadas indicaron que la calidad del producto hortícola constituye la principal característica, seguida por el precio (Cuadro 6).

Otra variable analizada fue si efectivamente el entrevistado encontraba la característica definida como la de mayor interés cada vez que realizaba las compras semanales de hortalizas, donde en el 48,9\% de los casos la respuesta fue que a veces existía la concordancia entre lo esperado y lo recibido y en otras ocasiones no era de esa manera (Cuadro 7). 
Cuadro 4. Media poblacional y límites al 95\% de confianza para el consumo semanal por producto hortícola. Buenos Aires, Golfito, Osa, Coto Brus, Corredores. Costa Rica. Abril-agosto. 2011.

\begin{tabular}{|c|c|c|c|c|c|}
\hline \multirow[t]{2}{*}{ Hortaliza } & \multirow[t]{2}{*}{ Unidad } & \multicolumn{3}{|c|}{ Consumo semanal en la población de estudio } & \multirow{2}{*}{$\begin{array}{c}\text { Valor por vivienda } \\
\begin{array}{c}\text { Valor máximo } \\
\text { encontrado }\end{array} \\
\end{array}$} \\
\hline & & Promedio & Límite inferior & Límite superior & \\
\hline Ajo & Cabeza (30 g aprox) & 98598 & 90511 & 106968 & 10 \\
\hline Albahaca & Rollo (400 g) & 90 & 39 & 142 & 3 \\
\hline Alfalfa & Paquete (200 g) & 51 & 0 & 107 & 9 \\
\hline Apio & Rollito (200 g) & 46767 & 41378 & 52156 & 11 \\
\hline Ayote sazón & Kilogramo & 8589 & 6.570 & 10608 & 10 \\
\hline Ayote tierno & Unidad & 22473 & 18652 & 26294 & 15 \\
\hline Berenjena & Unidad & 64 & 24 & 104 & 2 \\
\hline Brócoli & Cabeza (500 g aprox) & 19956 & 17215 & 22698 & 6 \\
\hline Camote & Kilogramo & 8934 & 6777 & 11092 & 11 \\
\hline Cebolla & Kilogramo & 70576 & 63925 & 77227 & 11 \\
\hline Cebollino & Rollo (400 g) & 1270 & 884 & 1655 & 4 \\
\hline Chayote & Unidad & 123248 & 103549 & 142947 & 44 \\
\hline Coliflor & Unidad & 17114 & 14699 & 19530 & 5 \\
\hline Chile dulce & Unidad & 151761 & 138944 & 164578 & 22 \\
\hline Culantro castilla & Rollo (50 g) & 85079 & 76759 & 93398 & 14 \\
\hline Culantro coyote & Rollo (50 g) & 45669 & 39210 & 52128 & 25 \\
\hline Elote & Unidad & 49871 & 36116 & 63626 & 100 \\
\hline Elote dulce & Unidad & 439 & 0 & 1039 & 100 \\
\hline Eneldo & Rollo (50 g) & 0 & 0 & 0 & 0 \\
\hline Espárrago & Kilogramo & 25 & 4 & 46 & 3 \\
\hline Espinaca & Rollo (400 g) & 2759 & 2077 & 3442 & 5 \\
\hline Hongos frescos & Paquete (500 g) & 7 & 0 & 15 & 2 \\
\hline Lechuga & Unidad & 79986 & 71871 & 88100 & 14 \\
\hline Minivegetales & Bandejas (500 g) & 808 & 0 & 2109 & 9 \\
\hline Nampí & Kilogramo & 6242 & 4742 & 7742 & 8 \\
\hline Orégano & Rollo (50 g) & 5381 & 4179 & 6582 & 7 \\
\hline Papa & Kilogramo & 83771 & 77040 & 90502 & 10 \\
\hline Pepino & Kilogramo & 54642 & 47795 & 61490 & 15 \\
\hline Perejil & Rollo (50 g) & 109 & 0 & 234 & 20 \\
\hline Plátano & Unidad & 320280 & 280543 & 360016 & 100 \\
\hline Rábano & Rollo (400 g) & 4458 & 3315 & 5601 & 8 \\
\hline Remolacha & Unidad & 13508 & 11268 & 15747 & 7 \\
\hline Repollo & Cabeza (750 g aprox) & 30630 & 26404 & 34855 & 11 \\
\hline Tiquizque & Kilogramo & 4283 & 3244 & 5322 & 6 \\
\hline Tomate & Kilogramo & 93852 & 84370 & 103335 & 22 \\
\hline Tomillo & Rollo (50 g) & 70 & 17 & 123 & 5 \\
\hline Vainica & Kilogramo & 16250 & 14240 & 18259 & 5 \\
\hline Yuca & Kilogramo & 27476 & 23701 & 31252 & 11 \\
\hline Zanahoria & Kilogramo & 24054 & 21074 & 27034 & 4 \\
\hline Zapallo & Unidad & 222 & 113 & 332 & 4 \\
\hline Zuquini & Unidad & 89 & 36 & 143 & 3 \\
\hline
\end{tabular}




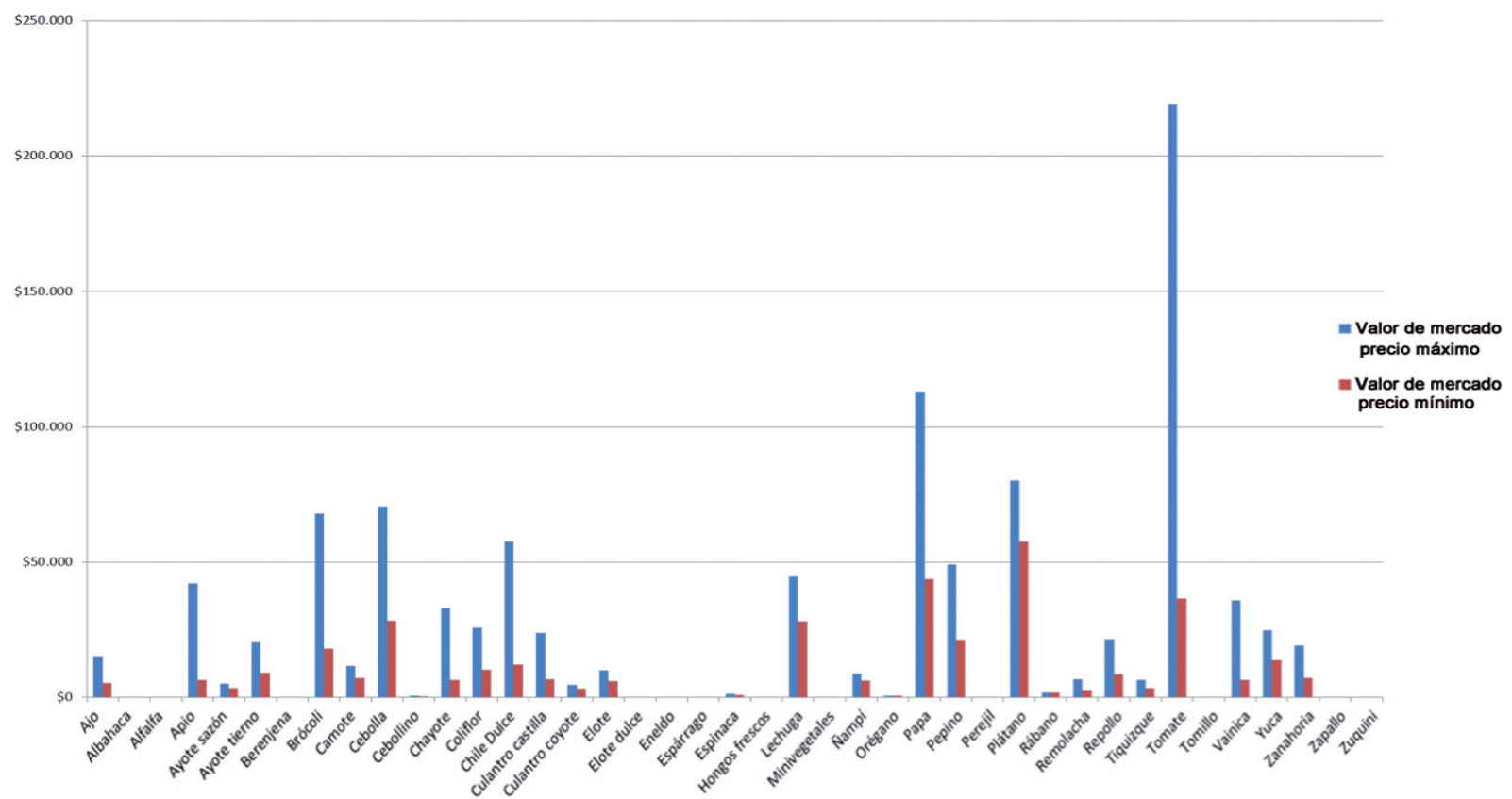

Figura 1. Valor semanal de mercado estimado para 41 hortalizas consumidas por las familias de la zona de estudio. Cantones de Buenos Aires, Osa, Golfito, Coto Brus y Corredores, provincia de Puntarenas, Costa Rica. Periodo del 1 de abril al 31 de agosto de 2011 .

Cuadro 5. Sitios de compra preferidos por las familias para adquirir las hortalizas de consumo semanal. Cantones de Buenos Aires, Osa, Golfito, Coto Brus y Corredores, provincia de Puntarenas, Costa Rica. Periodo del 1 de abril al 31 de agosto de 2011.

\begin{tabular}{llccc}
\hline Lugar preferido de compra & Frecuencia & Porcentaje & $\begin{array}{c}\text { Porcentaje } \\
\text { válido }\end{array}$ \\
\hline Válidos & Feria del agricultor & 35 & 8,9 & 12,7 \\
& Verdulerías & 56 & 14,2 & 20,4 \\
& Supermercados & 151 & 38,4 & 54,9 \\
& Otros lugares & 20 & 5,1 & 7,3 \\
& Autoconsumo & 13 & 3,3 & 4,7 \\
& Total & 275 & 70,0 & 100,0 \\
\hline \multirow{4}{*}{ Perdidos } & NR & 118 & 30,0 & \\
\hline \multicolumn{5}{c}{} \\
& Total & 393 & 100,0 & \\
\hline
\end{tabular}

Las respuestas del $44,5 \%$ de las familias consultadas sugieren que existe una entera concordancia entre las expectativas previas a la adquisición y lo que se encuentra semanalmente en los puntos de preferencia para realizar la compra, lo cual supone una satisfacción completa de los gustos y preferencias de este grupo poblacional. No obstante, se puede afirmar que el restante $55,5 \%$ de los consultados parece encontrar momentos de disconformidad en este sentido.

Para estimar la posibilidad de que en el futuro se presentaran cambios en el consumo actual de hortalizas, se realizó una pregunta orientada a conocer la respuesta del consumidor bajo el supuesto de que siempre existiera una oferta hortícola acorde con la totalidad de las expectativas 
Cuadro 6. Principal característica para decidir por la compra de hortalizas según la preferencia de los núcleos familiares. Cantones de Buenos Aires, Osa, Golfito, Coto Brus y Corredores, provincia de Puntarenas, Costa Rica. Periodo del 1 de abril al 31 de agosto de 2011.

\begin{tabular}{llccc}
\hline Principal característica & Frecuencia & Porcentaje & Porcentaje válido \\
\hline Válidos & Frescura & 280 & 71,2 & 71,2 \\
& Precio & 64 & 16,3 & 16,3 \\
& Tamaño & 10 & 2,5 & 2,5 \\
& Disponibilidad en el & 39 & 9,9 & 9,9 \\
mercado & & & 100,0 \\
\hline
\end{tabular}

Cuadro 7. Concordancia entre las expectativas del entrevistado según la principal característica para decidir por la compra de hortalizas. Cantones de Buenos Aires, Osa, Golfito, Coto Brus y Corredores, provincia de Puntarenas, Costa Rica. Periodo del 1 de abril al 31 agosto de 2011.

\begin{tabular}{lcccc}
\hline $\begin{array}{l}\text { Existencia de concordancias entre lo } \\
\text { esperado y lo recibido }\end{array}$ & Frecuencia & Porcentaje & Porcentaje válido \\
\hline Válidos & Siempre & 175 & 44,5 & 45,2 \\
& A veces & 192 & 48,9 & 49,6 \\
& Nunca & 20 & 5,1 & 5,2 \\
& Total & 387 & 98,5 & 100,0 \\
Perdidos & NS/NR & 6 & 1,5 & \\
\hline \multicolumn{2}{c}{} \\
\hline
\end{tabular}

previas por parte de la familia. Ante esta consulta, el $39,2 \%$ de los entrevistados afirmó que existiría mucha posibilidad de aumentar el consumo que semanalmente presenta su familia en la actualidad (Cuadro 8).

Cuadro 8. Posibilidad de aumentar las cantidades de compra del entrevistado en el caso de existir siempre una perfecta concordancia con la principal característica para decidir por la compra de hortalizas. Cantones de Buenos Aires, Osa, Golfito, Coto Brus y Corredores, provincia de Puntarenas, Costa Rica. Periodo del 1 de abril al 31 de agosto de 2011.

\begin{tabular}{lcccc}
\hline $\begin{array}{l}\text { Posibilidad de aumentar la } \\
\text { cantidad de compra }\end{array}$ & Frecuencia & Porcentaje & Porcentaje válido \\
\hline Válidos & Mucho & 154 & 39,2 & 39,2 \\
& Poco & 88 & 22,4 & 22,4 \\
& Más o menos & 108 & 27,5 & 27,5 \\
& NS/NR & 43 & 10,9 & 10,9 \\
\hline & 393 & 100,0 & 100,0 \\
\hline
\end{tabular}


Cuadro 9. Frecuencia de compras semanales de hortalizas para el núcleo familiar. Cantones de Buenos Aires, Osa, Golfito, Coto Brus y Corredores, provincia de Puntarenas, Costa Rica. Periodo del 1 de abril al 31 de agosto de 2011.

\begin{tabular}{lcccc}
\hline $\begin{array}{l}\text { Cantidad de veces que } \\
\text { compra hortalizas por semana }\end{array}$ & Frecuencia & Porcentaje & $\begin{array}{c}\text { Porcentaje } \\
\text { válido }\end{array}$ \\
\hline Válidos & 1 & 335 & 85,3 & 85,9 \\
& 2 & 36 & 9,2 & 9,2 \\
& 3 & 4 & 1,0 & 1,0 \\
& 5 & 1 & 0,3 & 0,3 \\
& 7 & 12 & 3,1 & 3,1 \\
& 9 & 1 & 0,3 & 0,3 \\
& 1 & 1 & 0,3 & 0,3 \\
& Total & 390 & 99,2 & 100,0 \\
\hline \multirow{4}{*}{ Perdidos } & NR & 3 & 0,8 & \\
\hline
\end{tabular}

No obstante lo anterior, un $22,4 \%$ de los entrevistados considera poco probable que aumente su consumo ante la ocurrencia de este supuesto.

También se realizaron consultas para conocer por la frecuencia de compra de productos hortícolas, donde el 85,3\% de los entrevistados afirmó que realiza solamente una compra semanal (Cuadro 9).

\section{LITERATURA CITADA}

Chiavenato, I. 2000. Introducción a la teoría general de la administración. 5 ed. McGraw-Hill Interamericana Editores S.A de C.V. Ciudad de México, México. $1056 \mathrm{p}$.

FAO (Organización de las Naciones Unidas para la Agricultura y la Alimentación) 2012. Qué es el Codex Alimentarius? (en línea). Roma. Consultado 15 feb. 2012. Disponible en ftp://ftp.fao.org/codex/ Publications/understanding/Understanding_ES.pdf

Gómez, M. 1998. Estadística descriptiva e inferencial. 3 ed. EUNED. San José, Costa Rica. 543 p.

INEC (Instituto Nacional de Estadística y Censo). 2000. IX Censo Nacional de Población y V de Vivienda. Instituto Nacional de Estadísticas y Censos, San José, Costa Rica. 80 p.
INEC (Instituto Nacional de Estadística y Censo). 2011. X Censo Nacional de Población y VI de Vivienda 2011: Cifras preliminares de población y vivienda. Instituto Nacional de Estadísticas y Censos, San José, Costa Rica. 46 p.

Kotler, P; Armstrong, G. 2003. Fundamentos de marketing. 6 ed. Pearson Educación. Ciudad de México, México. 680 p.

Mendenhall, W; Scheaffer, R; Ott, L. 2008. Elementos de muestreo. 6 ed. Thomson Editores. Madrid, España. $462 \mathrm{p}$.

Nicola, S; Fontana, E. 2010. Global horticulture: challenges and opportunities. Acta Hort. (ISHS) 856:49-54 (en línea). Consultado 9 nov. 2012. Disponible en http:// www.actahort.org/books/856/856_5.htm

PIMA (Programa Integral de Mercado Agropecuario). 2012a. Boletines de precios de venta del mayorista al minorista (en línea). Consultado 20 may. 2012. Disponible en http://www.pima.go.cr/

PIMA (Programa Integral de Mercado Agropecuario). 2012b. Tendencias del consumo de frutas, hortalizas y pescado en las familias de Costa Rica (en línea). Consultado 13 jun. 2012. Disponible en http://www.pima.go.cr/ Docs/Dedmdoc/Documento\%20Estudio\%20de\%20 tendencias\%202010-web.pdf

Solomon, M. 2008. Comportamiento del consumidor. 7 ed. Pearson Educación. Ciudad de México, México. 672 p. 